\title{
USO DE ESTIMULANTES DO SISTEMA NERVOSO CENTRAL POR ESTUDANTES DE SAÚDE DO SERTÃO DE PERNAMBUCO
}

\section{USE OF CENTRAL NERVOUS SYSTEM STIMULATORS BY HEALTH STUDENTS IN THE SERTÃO DE PERNAMBUCO}

\author{
Gustavo da Silva Cândido ${ }^{1} *$ João Paulo da Silva Teixeira ${ }^{2} *$ Larissa Gabrielle Torres $^{*}$ \\ Príncipe $^{3 *}$ Maria Viviane Mariano Terto ${ }^{*}$ Vitória Mayane Amorim Roque ${ }^{5}$ Viviane da \\ Silva Lima ${ }^{6}$ Gabriela Cavalcante da Silva ${ }^{7}$
}

\begin{abstract}
RESUMO
Introdução: Os psicoestimulantes são substâncias capazes de atuar no cérebro, produzindo efeitos ao modular a transmissão sináptica, estimulam ou inibem alguns neurotransmissores. Seus efeitos farmacológicos principais exercem função sobre o humor e estado de vigília, além de aumentar o estado de alerta e aprimoramento cognitivo. Eles têm sido usados por indivíduos que não possuem nenhum distúrbio neuropsiquiátrico ou cognitivo. Objetivo: Avaliar o uso de estimulantes do sistema nervoso central por estudantes de saúde do município de Serra Talhada - PE. Metodologia: Estudo transversal, com abordagem quantitativa com 325 estudantes de saúde da Faculdade de Integração do Sertão, Serra Talhada. Para a coleta de dados, utilizou-se um questionário auto aplicativo estruturado, via formulário Google. Resultados: Houve um alto índice de acadêmicos que se automedicam 71,1\% ( $\mathrm{n}=231)$. Dos 325 estudantes, 69,84\% ( $\mathrm{n}=227)$ relataram que já fizeram uso de algum psicoestimulante como hipnótico, Ginkgo biloba, ritalina (metilfenidato), bebida energética e cafeína para auxiliar na rotina dos estudos. Conclusão: $\mathrm{O}$ uso irracional de psicofármacos no âmbito acadêmico crescimento é crescente, e deve ser considerado problema de saúde pública, principalmente diante dos riscos de danos e efeitos adversos prejudiciais à saúde mental e física associados ao seu uso. Investigações devem ser desenvolvidas a fim de viabilizar o dimensionamento do problema, subsidiando ações de prevenção aos danos e dependência relacionados a esta classe de medicamento.
\end{abstract}

Palavras-chave: Saúde mental. Automedicação. Estimulantes do Sistema Nervoso Central.

\section{ABSTRACT}

Introduction: Psychostimulants are substances capable of acting on the brain, producing effects by modulating synaptic transmission, stimulating or inhibiting some neurotransmitters. Its main pharmacological effects have a role on mood and wakefulness, in addition to increasing alertness and cognitive improvement. They have been used by individuals who have no neuropsychiatric or cognitive disorders. Objective: To evaluate the use of central nervous system stimulants by health students in the municipality of Serra Talhada - PE. Methodology: Cross-sectional study, with a quantitative approach with 325 health students from the Faculty of Integration of the Sertão, Serra Talhada. For data collection, a selfstructured questionnaire was used, using the Google form. Results: There was a high rate of academics who self-medicate $71.1 \%(n=231)$. Of the 325 students, 69.84\% $(n=227)$ reported that they had already used some psychostimulant such as hypnotic, Ginkgo biloba, ritalin (methylphenidate), energy drink and caffeine to assist in the routine of studies. Conclusion: The irrational use of psychotropic drugs in the academic context is growing, and it should be considered a public health problem, especially in view of the risks of damage and adverse adverse effects on mental and physical health associated with its use. Investigations must be developed in order to make the problem sizing feasible, supporting actions to prevent damage and dependence related to this class of medication.

Keywords: Mental health. Self-medication. Central Nervous System Stimulants.

\footnotetext{
${ }^{1}$ Enfermeiro pela Faculdade de Integração do Sertão-FIS. Residente em Saúde Coletiva com ênfase em Agroecologia (Universidade de Pernambuco/Instituto Aggeu Magalhães). E-mail: gustavocandidogu@ gmail.com Orcid: https://orcid.org/0000-0002-3505-3634

${ }^{2}$ Enfermeiro pela Faculdade de Integração do Sertão-FIS. E-mail: joaopaulo77teixeira@ @mail.com Orcid:https://orcid.org/0000-0002-1282-9903

${ }^{3}$ Enfermeira pela Faculdade de Integração do Sertão-FIS. E-mail: gabriellyprincipe @ hotmail.com Orcid: https://orcid.org/0000-0002-3505-3634

${ }^{4}$ Enfermeira pela Faculdade de Integração do Sertão-FIS. E-mail: tertoviviane1998@ gmail.com Orcid:https://orcid.org/0000-0002-2432-8727

${ }^{5}$ Enfermeira pela Faculdade de Integração do Sertão-FIS. E-mail: vitoriamayaneamorim@ @outlook.com Orcid: https://orcid.org/0000-0003-0412-6973

${ }^{6}$ Farmacêutica Generalista pela Faculdade de Integração do Sertão-FIS. Esp. em Gestão Clínica do Medicamento pela Universidade Cruzeiro do SulUNICSUL. E-mail: limmavivianne@ gmail.com Orcid: https://orcid.org/0000-0003-0412-6973

Mestre em Ciências Farmacêuticas - UFPE. Doutora em Bioquímica e Fisiologia - UFPE. Docente da Faculdade de Integração do Sertão FIS. E-mail: gcavalcante1988@gmail.com Orcid: https://orcid.org/0000-0003-3390-6645
} 


\section{INTRODUÇÃO}

Os psicoestimulantes são substâncias que atuam no sistema nervoso central (SNC), produzindo efeitos ao modular a neurotransmissão sináptica. Em geral, seus efeitos farmacológicos principais de estimulante exercem função sobre o humor e estado de vigília, além de aumentar o poder de produtividade, permitindo maior estado de alerta e aprimoramento cognitivo. As principais substâncias utilizadas para essa finalidade são: cafeína, metilfenidato, modafinil, variadas bebidas energéticas, anfetaminas, dentre outras ${ }^{(1)}$.

A automedicação é entendida como iniciativa de um indivíduo ingerir substâncias de ação medicamentosa sem orientação ou acompanhamento de um profissional de saúde habilitado. $\mathrm{O}$ indivíduo pode ter acesso ao medicamento tanto comprando-o sem a devida prescrição, ou usar receituário vencido, dentre outras formas. $\mathrm{O}$ uso de medicamentos por conta própria é um hábito frequente e que transcende os conhecimentos técnico-científicos, objetivando solucionar algum problema relacionado à saúde, seja através do tratamento para cura ou alívio de algum incômodo, por muitas vezes sem o diagnóstico estabelecido de forma devida ${ }^{(2,3)}$.

A automedicação com metilfenidato como prática de melhoramento cognitivo farmacológico faz parte da vida de muitos dos estudantes universitários. Isso carece de ser compreendido e encarado como problema de saúde pública devido à exposição a riscos de danos e efeitos adversos associados à saúde física e mental de acadêmicos dos cursos de saúde ${ }^{(4)}$

A prática de consumo de estimulantes cerebrais entre estudantes universitários da área da saúde deveria ser menos prevalente, pois esses indivíduos têm acesso a disciplinas as quais abrangem conhecimento científico a temática. Porém, o acúmulo de conhecimento geral do indivíduo, somado a experiência de vida, pode levar a autoconfiança para se automedicar, como ocorre entre alguns profissionais de saúde ${ }^{(5)}$.

Correlacionado ao uso indevido e por vez irracional está o problema da dependência química, caracterizado pelo uso contínuo, exagerado como também a tolerância a algum tipo de substância, onde os meios social e cultural também podem excitar tal prática, gerando consequências negativas. Um profissional de saúde dependente de algum tipo de droga, por exemplo, expõe seus pacientes a maiores riscos, pois suas habilidades e capacidade de julgamento podem estar prejudicadas ${ }^{(6)}$.

Os componentes presentes nas bebidas energéticas podem levar a alguns efeitos colaterais como: câimbras, insuficiência renal, dependência, insuficiência hepática, aumento de peso, insônia, taquicardia, desidratação, agitação excessiva. É por isso que se deve ficar atento a frequência da ingestão dessas 
bebidas e prestar atenção nos sinais do corpo, pois pode haver intoxicação ${ }^{(7)}$. $\mathrm{O}$ metilfenidato em curto prazo pode acarretar em: redução de apetite e insônia, dor abdominal, hipertensão arterial e cefaleia. Em longo prazo os efeitos de maior importância foram dependência, problemas psicológicos e efeitos cardiovasculares ${ }^{(8)}$.

Diante do exposto, frente a essa realidade e a carência de informações nesse contexto, torna-se relevante avaliar o uso de estimulantes do sistema nervoso central por estudantes de saúde do município de Serra Talhada - PE. Cabe salientar que esse tipo de estudo pode ser útil para identificar a premência de intervenções para conscientizar essa população a adotar hábitos seguros e saudáveis no que tange os estudos e a vida profissional. Logo, necessita-se de mais estudos e ações sobre essa temática pela complexidade que envolve a saúde mental neste contexto político-social apresentado e pela necessidade de abordagem multiprofissional, este projeto apresenta uma proposta de intervenção no cuidado e preservação, a fim de proteger a saúde metal dos que podem se prejudicar pela prática errônea da automedicação.

\section{MATERIAIS E MÉTODOS}

Este estudo transversal, com abordagem do tipo quantitativa foi executado através da aplicação on-line do instrumento de coleta aos estudantes da área da saúde da Faculdade de Integração do Sertão - FIS, rua João Luiz de Melo, 2110 - Tancredo Neves, Serra Talhada - PE, 56909-205, durante os meses de agosto a outubro de 2020 na, mediante devida autorização dos dirigentes da instituição e pós deliberação do comitê de ética, através do protocolo número CAAE: 36968820.6.0000.8267 e parecer: 4.357.338.

A instituição FIS está localizada Município de Serra Talhada, localizado no Sertão Pernambucano, a uma distância de 415 $\mathrm{km}$ da capital pernambucana, Recife. O município possui uma população estimada de 86.350 mil habitantes (2019) conforme panorama do Instituto Brasileiro de Geografia e Estatística (IBGE, 2019). Esta conta com 1.305 (mil trezentos e cinco) alunos com matricula ativa do núcleo de saúde, semestre 2020.2. A amostra foi composta por estudantes de área de saúde contemplando sete cursos, os quais são: Enfermagem, Fisioterapia, Farmácia, Educação Física, Nutrição, Odontologia e Psicologia, estatisticamente a amostra deste trabalho contou com total de 325 (duzentos e oitenta e um) estudantes de graduação.

Foram inclusos neste estudo, acadêmicos devidamente matriculados na instituição de ensino, na área da saúde, indivíduos de ambos os sexos, que aceitaram participar da pesquisa espontaneamente, mediante assinatura do Termo de Consentimento Livre e Esclarecido - TCLE, o 
qual foi disponibilizado virtualmente, $\mathrm{n}$ a primeira secção do formulário Google que também continha o instrumento de coleta. Foram excluídos da pesquisa os acadêmicos menores de 18 anos, bem como aqueles que, por ventura, são portadores de Transtorno de Déficit de Atenção e Hiperatividade (TDAH).

As variáveis contempladas no instrumento de coleta foram: sexo, faixa etária, período do curso de graduação, conhecimento sobre efetividade dos psicoestimulantes, a frequência do uso, os efeitos colaterais apresentados, se houve indicação por profissional de saúde, quais os tipos de estimulantes cerebrais que mais consomem, se têm conhecimento sobre os riscos que essa prática pode trazer. Tal instrumento de coleta como também o TCLE, confeccionados em formato digital, foram disponibilizados aos alunos através dos grupos de WhatsApp das turmas de alunos da instituição. $\mathrm{O}$ software utilizado foi GraphPad Prism versão 8.0 foi utilizado para tabulação de estatísticas analíticas e para apresentação dos dados. Os resultados foram dispostos pelo programa, utilizando-se a frequência de (\%) e média.

\section{RESULTADOS E DISCUSSÃO}

Dos 325 questionários coletados, $26,5 \%(n=86)$ dos alunos possuem entre 1820 anos, $(66,12)(\mathrm{n}=215)$ entre 21-30 anos, 6,2\% ( $n=20)$ entre 31-40 anos, 0,9\% ( $n=3)$ entre 41-50 anos e no grupo acima de 51 anos foi registrado $0,3 \% \quad(n=1)$. Os voluntários concentraram-se entre 21-30 anos 66,15\% $(\mathrm{n}=215) \quad$ (Gráfico 1A). O sexo dos entrevistados foi mais prevalente para o feminino, representando 76,9/\% $(\mathrm{n}=250)$ dos estudantes, já o sexo masculino representou 23,1\% (n=75) (Gráfico 1B).

Em estudo recente 200 questionários foram aplicados a universitários da Universidade Federal do Rio Grande - Rio Grande do Sul, o sexo feminino representou $60,2 \%(\mathrm{n}=118)$ dos estudantes, e a idade média foi de 23,3 anos (DP = 4,1), com 46,2 $\%(\mathrm{n}=91)$ dos estudantes situados na faixa de 22 a 25 anos. Já na Universidade Federal de Minas Gerais foram incluídos 378 estudantes (1). A média de idade dos estudantes respondentes foi de 27,9 anos $(\mathrm{DP}=8,2)$ e as mulheres também representaram a maioria da $\operatorname{amostra}(64 \%)^{(4)}$. 
Gráfico 1 - Faixa etária (A) e gênero (B) dos estudantes de saúde da Faculdade de Integração do Sertão, Serra Talhada, 2020.

A

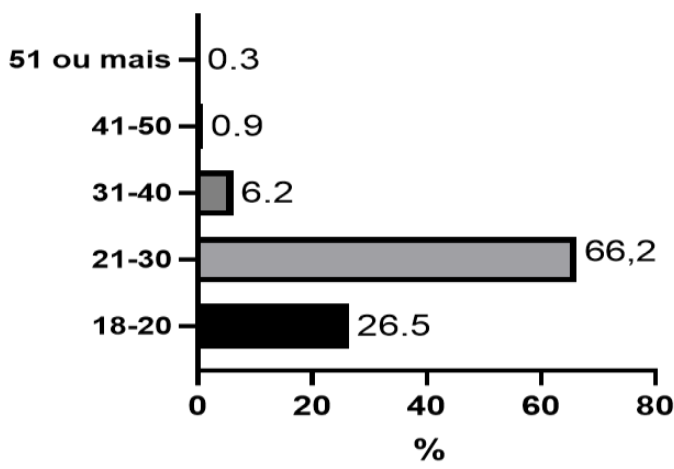

Fonte: Os autores

Em relação ao estado civil dos alunos entrevistados, $87,69 \%(\mathrm{n}=285)$ declarou estar solteiro, representando a maioria. Pessoas casadas representam 9,23\% $(n=30)$ das que participaram da pesquisa, além de $2,77 \%$ $(n=9)$ divorciados e apenas $0,31 \% \quad(n=1)$ viúvo. O (gráfico 2) mostra a distribuição dos estudantes participantes do estudo nos seus respectivos cursos, sendo que prevaleceu a
B

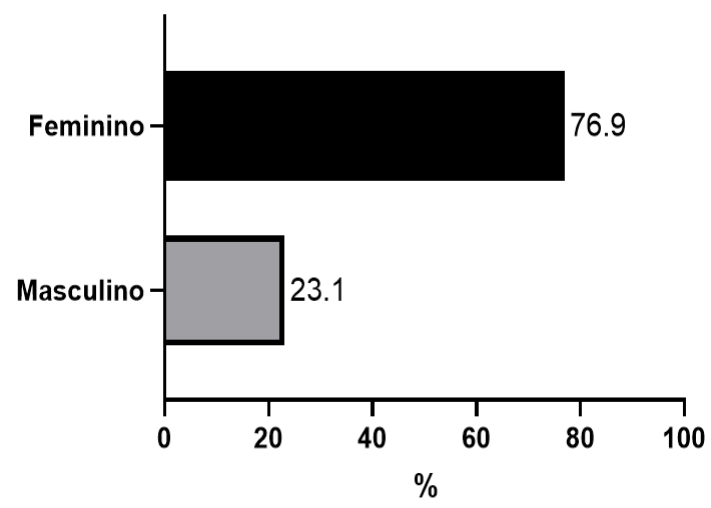

participação dos acadêmicos de farmácia $30,15 \%(n=98)$ e enfermagem $28,31 \%(n=92)$.

Em instituição de ensino superior do Distrito Federal, 144 estudantes participaram de uma pesquisa. Essa pesquisa foi realizada com estudantes de vários cursos superiores, sendo que os estudantes do curso de farmácia tiveram percentual de maior participação, totalizando $48 \%(n=69)$ e enfermagem $16 \%$ $(\mathrm{n}=23)^{(9)}$.

Gráfico 2 - Curso dos estudantes de saúde entrevistados da Faculdade de Integração do Sertão, Serra Talhada, 2020.

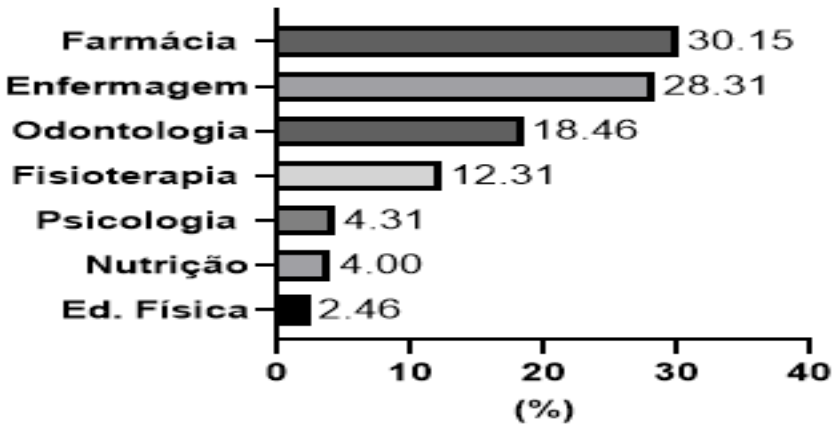

Fonte: Os autores 
Conforme ilustrado no gráfico 3, $71,1 \%(\mathrm{n}=231)$ dos estudantes responderam que costumam usar medicamento sem prescrição de um profissional de saúde. Tal resultado corrobora com estudo realizado com estudantes de fisioterapia de uma instituição privada - Centro Universitário do Triângulo (UNITRI) de Minas Gerais, onde detectou-se que $94,43 \%$ dos entrevistados afirmaram que já utilizaram ou compraram medicamentos sem receita médica. Dentre os principais motivos/doenças que levam à automedicação, sendo que $31,30 \%$ responderam a dor de cabeça, sendo que as outras maiores frequências obtidas são em doenças tais como resfriado, febre e infecção de garganta, todavia sempre acompanhado da dor de cabeça ${ }^{(10)}$.

Gráfico 3 - Entrevistados que costumam usar medicamento sem prescrição, Faculdade de Integração do Sertão, Serra Talhada, 2020.

Fonte: Os autores

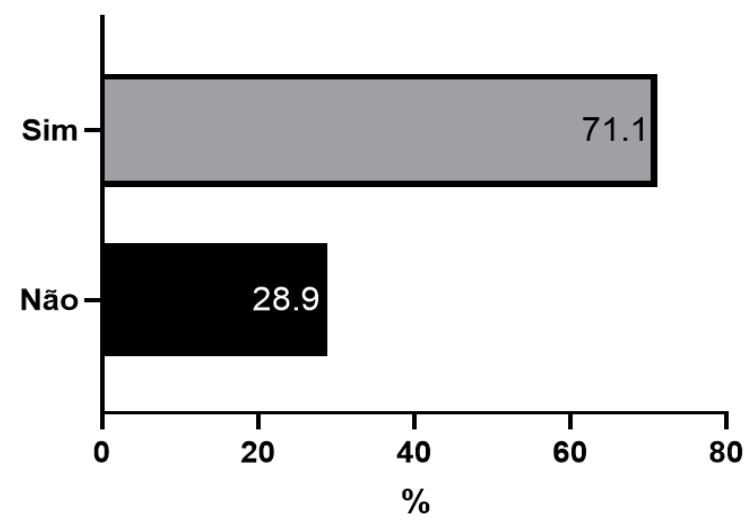

O Brasil ocupa a quinta posição entre os países mais consumidores de medicamentos, configurando o primeiro lugar na América Latina. Utilizar medicamento sem prescrição, como autocuidado, pode ter resultados benéficos para o indivíduo, como: a melhora de sintomas, resolução de problemas de saúde, mas outras vezes pode ser prejudicial à saúde do indivíduo, podendo mascarar problemas de saúde, causar intoxicações, reações adversas, interações medicamentosas, desenvolvimento de resistência, entre outros ${ }^{(11)}$.
Em relação ao grau de conhecimento sobre psicoestimulantes, 11,38\% ( $\mathrm{n}=37)$ dos entrevistados responderam que possuem conhecimento amplo, 45,23\% $\quad(n=147)$ conhecimento razoável, 34,77\% $\quad(n=113)$ responderam que possuem conhecimento restrito, e 8,62\% (n=28) desconhece esse assunto (gráfico 4 A).

O uso indiscriminado desses fármacos tem sido discutido cada vez mais em diversos países, pois, na contemporaneidade o ritmo acelerado e estilo de vida levam a população, principalmente estudantes e profissionais da 
saúde, a vivenciarem situações estressantes e dificultosas. Uma das opções adotadas referese ao uso de substâncias psicoativas (SPA), como tentativa de aliviar ou diminuir o estresse e cansaço $^{(12)}$.

Gráfico 4 - Grau de conhecimento sobre psicoestimulantes (A) e uso de algum medicamento para auxiliar no estudo (B) pelos estudantes de saúde entrevistados da Faculdade de Integração do

Sertão, Serra Talhada, 2020.

A

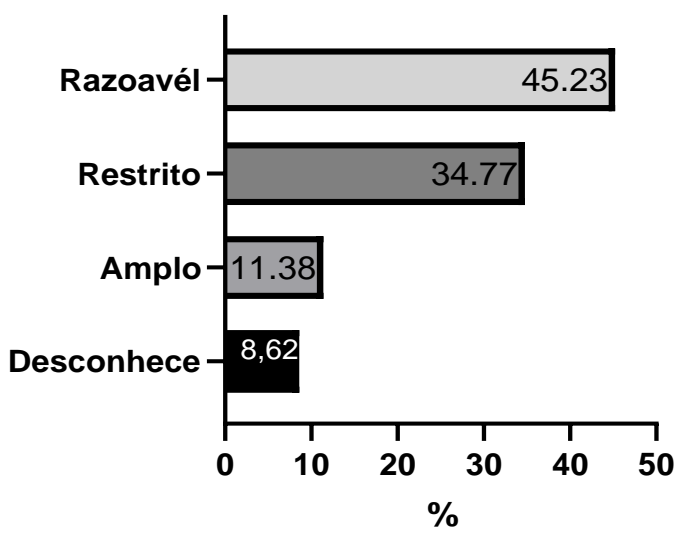

Fonte: Os autores

A prevalência de uso de medicamento para auxiliar no estudo de forma geral, foi de 28,3\% (n=92) (Gráfico 4B). 69,84\% ( $\mathrm{n}=227)$ dos estudantes relataram que já fizeram uso de alguma substância estimulante do sistema
B

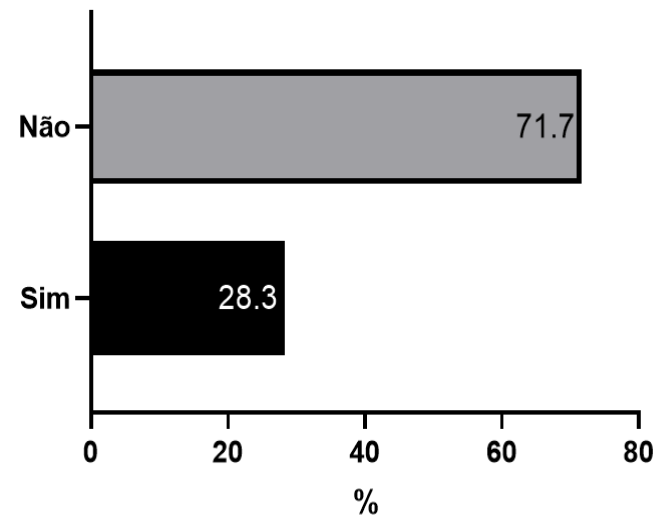

nervoso central listada no questionário, como ansiolítico, antidepressivo, hipnótico, Ginkgo biloba, ritalina (metilfenidato), bebida energética e cafeína, como descritas no gráfico (Gráfico 5).

Gráfico 5 - Descrição do uso de substâncias psicoestimulantes por estudantes de saúde entrevistados da Faculdade de Integração do Sertão, Serra Talhada, 2020.

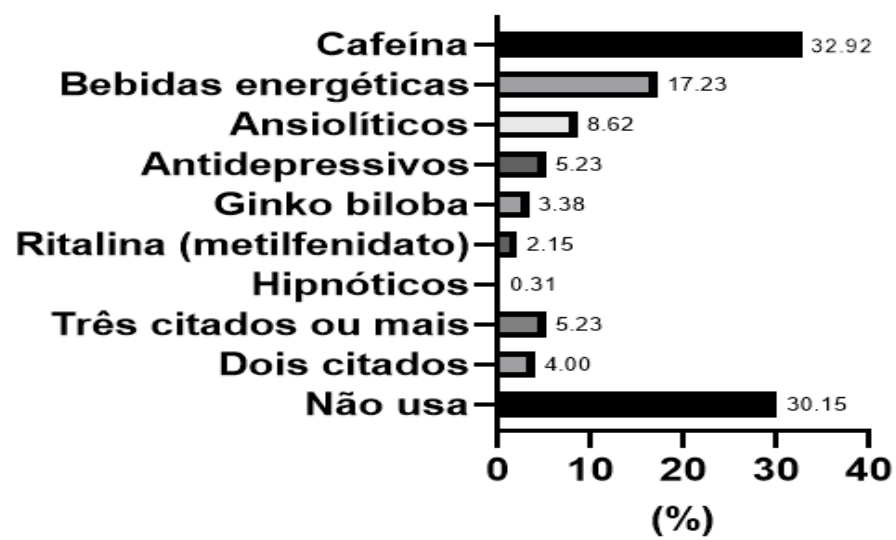

Fonte: Os autores 
Em se tratando da frequência do uso

dos estimulantes, $\quad 34,8 \% \quad(\mathrm{n}=113)$ responderam que faz uso diariamente, $21,8 \%$ $(\mathrm{n}=71)$ esporadicamente (Gráfico 6A).
Encontrou-se maior percentual de uso de bebidas energéticas para auxiliar no estudo $76 \%$ ( $\mathrm{n}=247)$, como açaí, café, energético ou refrigerante, por exemplo (Gráfico 6B).

Gráfico 6 - Frequência do uso de estimulante cerebral (A) e uso de bebida energética estimulante cerebral (B) pelos entrevistados da Faculdade de Integração do Sertão, Serra Talhada, 2020.

A

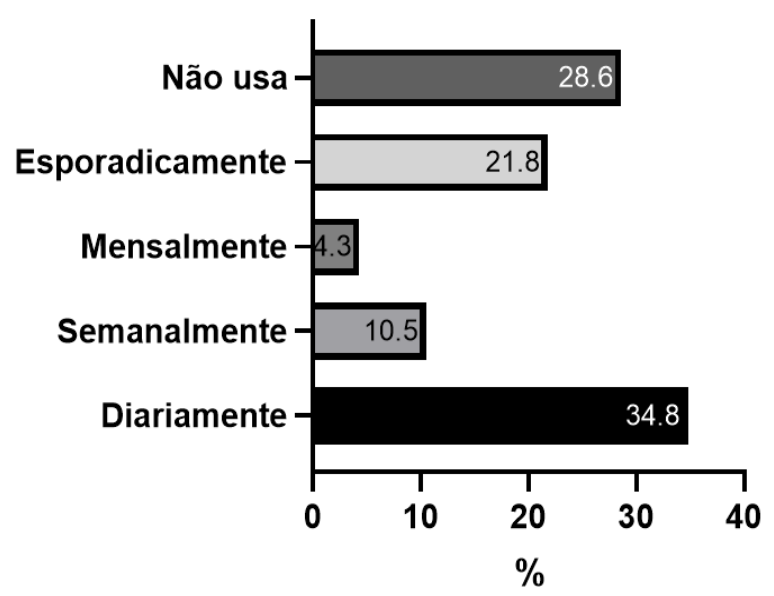

Fonte: Os autores

A cafeína é a droga psicoativa mais utilizada no mundo, configurando o principal componente das bebidas energéticas bastante consumidas pelos jovens estudantes brasileiros ${ }^{(13)}$. Estudo com 116 universitários mostrou que a cafeína é o psicoestimulador utilizado com maior frequência pelos estudantes, representando $55,66 \%$ do uso entre esses acadêmicos e dentre eles, $95,45 \%$ não possuem prescrição para adquirir e utilizar algum tipo de estimulante cerebral. Apontou-se ainda que o café, o chá mate, o guaraná, termogênicos e refrigerantes feitos à base de cola são alguns tipos de estimulantes mais utilizadas pelos universitários ${ }^{(14)}$.

\section{B}

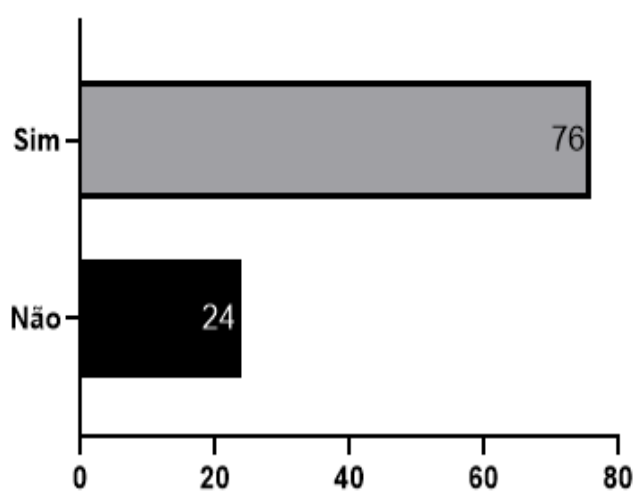

(\%)

Evidenciou em uma faculdade privada do Distrito Federal, em levantamento com estudantes dos cursos de Biomedicina, Enfermagem, Farmácia e Nutrição que de um total de 400 entrevistados 19,5\% (78 entrevistados) afirmaram que já fizeram uso de algum medicamento para auxiliar na rotina dos estudos, como o pó de guaraná, sendo então a substância mais citada (49\%), seguido de Ginkgo biloba (35\%). Do total de entrevistados, $6,0 \%$ relataram o uso de metilfenidato, e dentre esses, somente $16,7 \%$ possuíam o diagnóstico médico de TDAH ${ }^{(15)}$.

Os resultados do estudo mostraram que, dentre os estudantes que declararam que já utilizaram ou fazem uso de algum 
estimulante do SNC, o metilfenidato retrata 2,15\% (n=7) das respostas (Gráfico 5). Outro estudo realizado com discentes da Universidade Federal de Minas Gerais afirma que o consumo de metilfenidato, independente do momento da vida, foi relatado por 37 estudantes (37/378; 9,8\%). Dentre estes, $22(22 / 37 ; 59 \%)$ declararam ter feito seu uso para neuroaprimoramento $(22 / 378 ; 5,8 \%)$. A prevalência de consumo recente de metilfenidato para neuroaprimoramento foi estimada em 2,4\% $(9 / 378)^{(4)}$.

Com relação às áreas do conhecimento, os estudantes de exatas e humanas formaram um grupo mais propenso a utilizar o metilfenidato para neuroaprimoramento, independente do momento da vida. Por outro lado, um estudo quantitativo observacional do tipo transversal feito com 200 estudantes de graduação em Medicina, mostrou que a prevalência de uso de substâncias estimulantes foi de 57,5\%, sendo que $51,3 \%$ destes começaram a usá-las durante a faculdade. $\mathrm{O}$ uso de psicoestimulantes no momento da pesquisa teve prevalência de 52,3\%, valendo destacar que $16,6 \%$ dos estudantes consumiam mais de uma substância psicoestimulante ${ }^{(1)}$.

As substâncias mais consumidas foram bebidas energéticas $(38,0 \%)$ e cafeína mais de cinco vezes por semana $(27,0 \%)$. Os principais motivos alegados para o consumo de estimulantes foram compensar a privação de sono $(47,4 \%)$ e melhorar raciocínio, atenção e/ou memória (31,6\%). Numa pesquisa quantitativa descritiva e exploratória realizada com 44 estudantes de enfermagem do Centro de Ensino Superior de Valença, localizado no município de Valença, 2,3\% $(n=1)$ dos entrevistados afirmaram utilizar tranquilizantes e ansiolíticos diariamente, $4,5 \%(n=2)$ menos de 1 vez por semana, $2,3 \%$ $(n=1) 2$ ou 3 vezes por semana e 11,4\% $(n=5)$ utilizaram nos últimos 3 meses antecessores ao questionário. Anfetamina foi utilizada nos últimos 3 meses por $2,3 \% \quad(n=1)$, antidepressivos diariamente por $4,5 \%(n=2)$ e nos últimos 3 meses $4,5 \%(n=2)$ fizeram uso (6).

Dentre os principais benefícios, aquele motivo mais apontado pelos participantes da pesquisa para o consumo de estimulantes cerebrais foi diminuição do sono, $(55 \%$, n=180) (Gráfico 7A). Já em relação aos sintomas pós uso, os entrevistados relataram dor de cabeça $(23,08 \%, \quad n=75)$, fadiga $(16,31 \%, \mathrm{n}=53)$, indisposição $(12 \%, \mathrm{n}=39)$, amnésia $(2,15 \%, n=7)$, pressão alta $(3,38 \%$, $\mathrm{n}=11)$, sudorese $(6,46 \%, \mathrm{n}=21)$, taquicardia $(10,46 \%, n=34) .4 \% \quad(n=13)$ tiveram dois sintomas citados e $6,46 \%(n=21)$ responderam que tiveram três ou mais sintomas (Gráfico 7B).

A maioria das manifestações clínicas consequentes do uso de anfetaminas, por exemplo, se devem à estimulação adrenérgica prolongada, principalmente em SNC e 
cardiovascular. Estão presentes, desde manifestações de agitação, sudorese, midríase, náuseas, dor abdominal, hipertensão, taquicardia e cefaleia, até manifestações mais graves, decorrentes do uso de altas doses, como hipertermia grave (> $40^{\circ} \mathrm{C}$ ), desidratação, arritmias, hipertensão grave, infarto agudo do miocárdio, vaso espasmo, acidente vascular cerebral, morte súbita, pneumotórax, psicose, convulsão, colite isquêmica, rabdomiólise, insuficiência hepática, delírios, paranoia, dentre outras ${ }^{(16)}$.

Gráfico 7- Benefícios do uso de estimulante cerebral (A) e sintomas pós uso de estimulantes cerebrais (B) apresentados pelos entrevistados da Faculdade de Integração do Sertão, Serra Talhada, 2020.

A

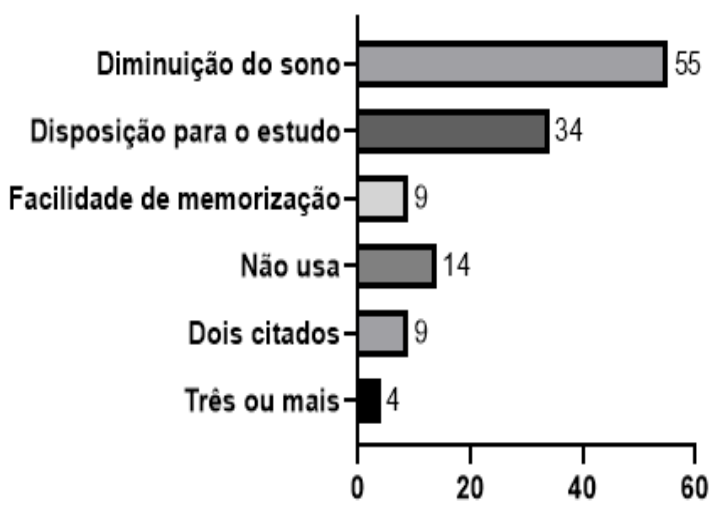

$(\%)$

Fonte: Os autores

Nenhuma substância estimulante do SNC é segura quando usada de forma irracional e sem supervisão, já que podem apresentar algum tipo de prejuízo ao usuário e sobretudo podem causar dependência. A crescente busca pelo aperfeiçoamento cognitivo farmacológico (ACF) tem se tornado um fator preocupante, já que há utilização banal, inclusive abusiva e sem controle pela população ${ }^{(17)}$.

Os eventos adversos medicamentosos são definidos como lesões derivadas do uso de medicações. Esse termo possui variadas
B

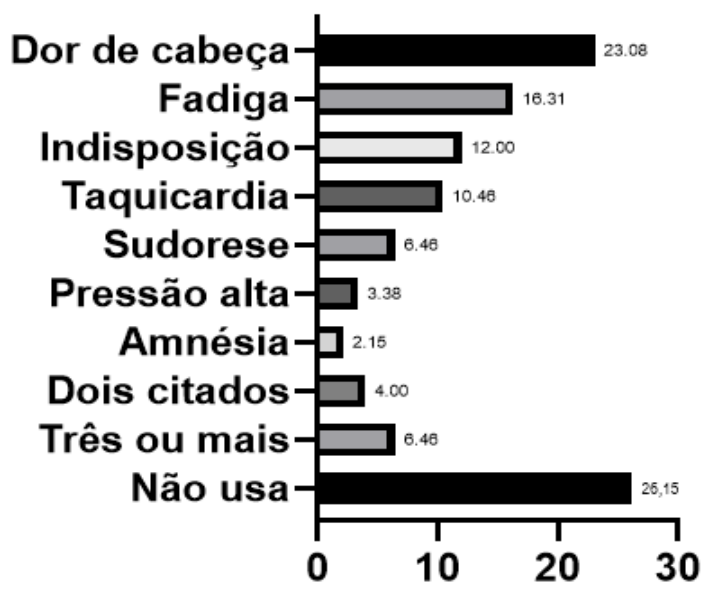

(\%)

definições, incluindo danos causados por medicamentos em uma dosagem usual ou em uma dosagem incomum, assim como incluem danos decorrentes da redução da dose e descontinuação da terapia medicamentosa ${ }^{(18)}$. Em longo prazo estimulantes cerebrais podem gerar dependência, a qual leva tanto ao sofrimento físico, quanto psicológico, e por vezes atrapalha as atividades cotidianas das pessoas levando a dificuldade de concentração e ansiedade. A dependência pode ter características como a tolerância, abstinência e falta de interesse em atividades 
importantes em razão do uso da substância.

Tolerância a uma droga é o que ocorre após repetidas e excessivas exposições entre a droga e o organismo, ou seja, ocorre a diminuição do efeito da droga, necessitando de doses maiores da substância para que o organismo corresponda da mesma forma e intensidade ${ }^{(17) .}$

\section{CONCLUSÃO}

Psicoestimulantes, embora promovam concentração, disposição, atenção e energia, também acabam por modificar o estado de humor e vigília dos usuários, de modo a elevar a pressão arterial, alterar a frequência cardíaca, entre outros efeitos danosos a saúde mental. Assim, é possível afirmar que os objetivos do presente trabalho foram alcançados. O uso excessivo e irracional de psicofármacos no âmbito acadêmico se encontra em crescimento e fomentar a racionalidade é uma tarefa complexa, pois se relaciona com aspectos sociais, econômicos, educativos e clínicos. Investigações devem continuar sendo realizadas para viabilizar o dimensionamento otimizado do problema, para que assim se desenvolva a prevenção aos danos com o também a dependência, episódios de insônia e psicoses, déficit na atenção e cognição.

\section{REFERÊNCIAS}

1. Morgan HL. et al. Consumo de Estimulantes Cerebrais por Estudantes de Medicina de uma Universidade do Extremo Sul do Brasil: Prevalência, Motivação e Efeitos Percebidos. Revista brasileira de educação médica., Rio de Janeiro, 2017; 41(1):102-9.

2. Bernardes HC. et al. Perfil epidemiológico de automedicação entre acadêmicos de medicina de uma universidade pública brasileira. Brazilian Journal of Health Review, 2020; 3 (4) :8631-8643.

3. Rios MF. et al. Perfil da automedicação dos alunos de uma escola técnica do Sul de Minas Gerais. Revista da Universidade Vale do Rio Verde, 2013; 11(2): 420-31.

4. Cândido RCF. et al. Prevalência e fatores associados ao uso de metilfenidato para neuroaprimoramento farmacológico entre estudantes universitários. Einstein (São Paulo), São Paulo, 2020; 18: 4720-4745.

5. Rodrigues AP. et al. A prática da automedicação em acadêmicos do curso de fisioterapia de uma instituição de ensino superior privada. e-RAC, 2015; 5(1): 1-23.

6. Neves FB. et al. Uso de substâncias psicoativas lícitas por estudantes de enfermagem. Revista Interdisciplinar de Direito, 2017; 10(2): 1-16.

7. Evangelista LN. As consequências da privação do sono e os efeitos do uso indiscriminado de psicoestimulantes. 2018. 48 f., il. Trabalho de Conclusão de Curso (Bacharelado em Farmácia) - Universidade de Brasília, Brasília.

8. Santana LC. et al. Consumo de Estimulantes Cerebrais por Estudantes em Instituições de Ensino de Montes Claros/MG. Rev. bras. educ. med., 2020; 44(1): 1-36.

9. Toledo JOD. et al. O uso da ritalina ${ }^{\circledR}$ (METILFENIDATO) para o desempenho acadêmico. Revista JRG De Estudos Acadêmicos, 2019; 2(5): 283-94. 
10. Rodrigues AP, Boaventura CDM, Magazoni VS; Cardoso-Filho GMA prática da automedicação em acadêmicos do curso de fisioterapia de uma instituição de ensino superior privada. e-RAC, 2015; 5(1): 20-35.

11. Iuras A. et al. Prevalência da automedicação entre estudantes da Universidade do Estado do Amazonas (Brasil). Revista Portuguesa de Estomatologia, Medicina Dentária e Cirurgia Maxilofacial, 2016; 57(2): 104-11.

12. Luna ISD. et al. Consumo de psicofármacos entre alunos de medicina do primeiro e sexto ano de uma universidade do estado de São Paulo. In: Colloquium Vitae. 2018; 1(2): 228.

13. Lino MFS, Silva CM. Bebidas energéticas: uma questão educacional/Energy drinks: an educational issue. Brazilian Journal of Development, 2019, 5(6): 4483-92.

14. Silveira VI. et al. Uso de psicoestimulantes por acadêmicos de medicina de uma universidade do Sul de Minas Gerais. Revista da Universidade Vale do Rio Verde, 2015; 13(2): 186-92.

15. Affonso RDS. et al. O uso indiscriminado do cloridrato de metilfenidato como estimulante por estudantes da área da saúde da faculdade anhanguera de brasília (FAB). Infarma Ciências Farmacêuticas. 2016; 28(3):166-72.

16. Hernandez EMM. et al. Manual de toxicologia clínica: orientações para assistência e vigilância das intoxicações agudas. 2017. In: Manual de toxicologia clínica: orientações para assistência e vigilância das intoxicações agudas.

17. Tsuda CA, Christoff AO. Avaliação do padrão de uso de estimulantes em uma faculdade de Curitiba-PR. Cadernos da Escola de Saúde, 2015; 1(13): 116-32.

18. Ayani N. et al. The epidemiology of adverse drug events and medication errors among psychiatric inpatients in Japan: the JADE study. BMC psychiatry, 2016; 16(1): 1-303.
Submissão: 2021-04-30

Aprovado: 2021-07-28 